\title{
Geschlossene Reinigung mit Methylenchlorid
}

\author{
Verunreinigte Rührwerksbehälter werden in der Quarzkomposit-Verarbeitung meist mit \\ Methylenchlorid im offenen Prozess manuell gereinigt. Ein neues Verfahren ermöglicht nun die \\ Reinigung im geschlossenen System, wodurch die Produktivität gesteigert, die Arbeitssicherheit \\ erhöht und die Umweltbelastung verringert wird.
}

Bei der Verarbeitung von Quarzkomposit werden Quarz, Harz, Farbe sowie Glasbruchstücke in Rührwerksbehältern gemischt, um daraus später unter hohem Druck Quarzkomposit-Kunststein für zum Beispiel Arbeitsplatten, Spülen oder Waschtische herzustellen. Bislang wurden die hartnäckigen, abrasiven Quarzkompositreste meist mit dem organischen Lösemittel Methylenchlorid (MC) gereinigt.

Methylenchlorid dient unter anderem als wirkungsvolles Abbeizmittel für Lacke, als Entfettungsmittel sowie als Lösungsmittel für Harze, Fette, Kunststoffe und Bitumen und kommt beispielsweise zur Entfernung von Verguss- und Gießharzen sowie zur Reinigung der Misch- und Dosieranlagen zum Einsatz. Da MC anlagentechnisch schwierig $\mathrm{zu}$ handhaben ist - der Siedepunkt liegt zum Beispiel unter $40^{\circ} \mathrm{C}$ - werden die Rührwerksbehälter manuell durch Facharbeiter von hartnäckigen Restprodukten befreit. Dazu füllen sie die Mischbehälter für mehrere Stunden mit
Methylenchlorid und spritzen sie dann per Hochdrucklanze ebenfalls mit MC aus. Bei unsachgemäßer Handhabung oder falscher Schutzausrüstung besteht jedoch das Risiko einer Gefährdung für Mensch und Umwelt.

\section{Produktivität steigern und Arbeitsplatzsicherheit erhöhen}

EVT Eiberger Verfahrenstechnik hat daher ein Verfahren und eine passende Sonderanlage entwickelt, um Methylenchlorid im geschlossenen System zu handhaben. Diese anwenderspezifische Sonderanlage ist nicht für das klassische Reinigen und Entfetten von Bauteilen in Arbeitskammern ausgelegt. Vielmehr werden die Mischbehälter in eine maßgefertigte Vorrichtung eingebracht und verriegelt. Durch eine spezielle Spritzlanze lässt sich das Lösemittel in den Behälter einspritzen. Ein Motor treibt das Rührwerk von extern an. So werden Behälter-Innenraum sowie alle Komponenten vollständig abgereinigt und unter messtechnischer Überwachung getrocknet.

Zum Abführen der Reinigungsmasse verzichtet EVT komplett auf Pumpensysteme, da Quarzkompositrückstände stark abrasiv sind. Der Transport innerhalb der Anlage wird mittels Druckdifferenzen gelöst. Die geschlossene Reinigung steigert die Produktivität und erhöht signifikant die Arbeitsplatzsicherheit. Darüber hinaus wird die Umwelt geschont und der Lösemittelverbrauch deutlich reduziert. //

\section{Kontakt}

EVT Eiberger Verfahrenstechnik GmbH

Sternenfels

info@evt-gmbh.eu

www.evt-gmbh.eu



Rührwerke und Behälter automatisiert statt manuell reinigen.

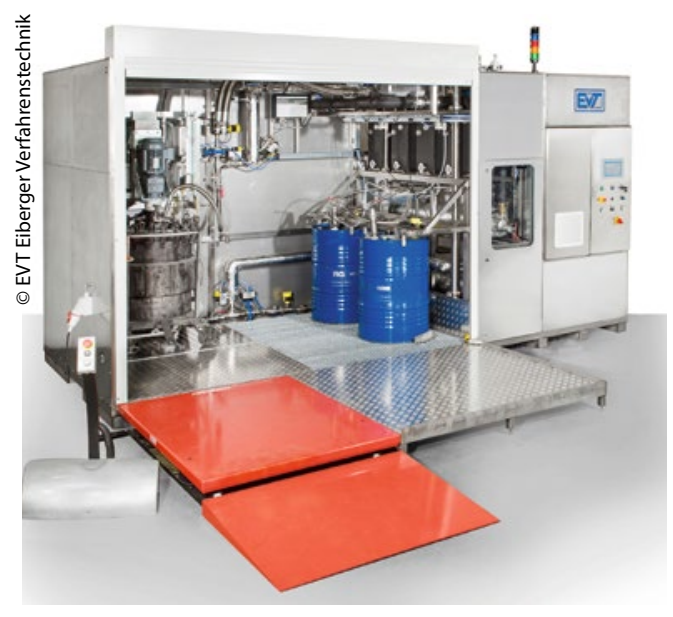

Die Sonderanlage zur geschlossenen Reinigung mit Methylenchlorid schützt Mitarbeiter und Umwelt. 